\title{
Countermeasures against Pattern Formation Phenomena of Thin Sheet Winder by Using Dynamic Absorbers*
} \author{
Takahiro RYU $^{* *}$, Kenichiro MATSUZAKI $^{* * *}$, Atsuo SUEOKA $^{* * *}$
and Hidetoshi MORITA \\ **Department of Mechanical and Energy Systems Engineering, Oita University, \\ 700 Dannoharu, Oita-shi, Oita 870-1192, Japan \\ Email: ryu@cc.oita-u.ac.jp \\ ***Kyushu University, \\ 744 Motooka, Nishi-ku, Fukuoka-shi, Fukuoka 819-0395, Japan \\ ****Sasebo National College of Technology, \\ 1-1 Okishincho, Sasebo-shi, Nagasaki 857-1193, Japan
}

\begin{abstract}
This paper deals with a countermeasure against pattern formation phenomena generated on thin sheet winder using dynamic absorbers. The thin sheet is widely used as paper, aluminum, etc. Pattern formation phenomena of the wound sheet sometimes occur in operation because of the viscoelastic characteristics of the sheet. The pattern formation phenomena lead to a fatal damage on the thin sheet. This problem is similar to the other pattern formation phenomena, for instance, polygonal deformations of the smoother roll of paper making machines and pattern formation of the elastic yarn of textile winding machines. The authors conducted experiment to generate the pattern formation using experimental apparatus, and theoretically analyzed to clarify the mechanism of the pattern formation phenomena considering the elastic deformation of the rolls. They also theoretically examined the countermeasure to suppress the phenomena by attaching some dynamic absorbers. The effectiveness of the tunable dynamic absorbers was confirmed by the theoretical analysis, and an example of the tunable dynamic absorber was introduced.
\end{abstract}

Key words: Self-Excited Vibration, Vibration of Rotating Body, Stability, Dynamic Absorber, Pattern Formation, Thin Sheet Winder

\section{Introduction}

Sheet material, such as paper, aluminum and plastic, is collectively called "thin sheet". A thin sheet winder consists of rolls. The thin sheet passes through the nip section under a load between the rolls, after which it is wound onto a rotating roll. During the winding process, the wound sheet periodically deforms, and strong vibrations are often generated in the machine. These factors can make continuous operation of the winder impossible, and they sometimes damage the quality of thin sheets ${ }^{(1)}$. Such phenomena frequently occur during the winding process of elastic yarn in a textile-winding machine, and in the smoother rolls of papermaking machine ${ }^{(2),(3)}$. These phenomena are called pattern formation phenomena.

Among the characteristics of thin sheet winders is the fact that the length of the rolls are long compared with their diameter, and their physical quantities - such as mass and inertia moment - vary in accordance with the increase in the thickness of the wound sheet. Hence, the elastic vibration of the rolls, the temporal variation of natural frequencies caused by the 
variation in system parameters, and the decrease of the rotating frequency need to be considered when analyzing pattern formation phenomena. In this paper, the authors conduct experiments to reproduce pattern formation using an experimental apparatus and theoretically analyze the system to clarify the generation mechanism of the pattern formation phenomena.

Recently, studies on measures to suppress pattern formation phenomena in hot levelers and rolling contact systems having viscoelastic characteristics were conducted using dynamic absorbers ${ }^{(5),(6)}$. In this study, the authors also theoretically analyzed the suppressive effect of dynamic absorbers on pattern formation phenomena in thin sheet winders, and based on this, they have proposed an optimal design for dynamic absorbers.

\section{Experimental Results}

Figures 1(a) and 1(b) show the outline of a thin sheet winder and the experimental apparatus, respectively. The thin sheet winder consists of a winding roll (henceforth "WR") and a nip roll (henceforth "NR"). The NR drives the WR by contact rolling through the thin sheet, and the thin sheet is wound onto the WR. The surface of the NR is covered with rubber that is harder than the thin sheet being processed. The WR and the NR are hollow cylinders, having outer diameters of $500 \mathrm{~mm}$ and $350 \mathrm{~mm}$, respectively, and both rolls are $8.8 \mathrm{~m}$ long. As the peripheral speed of the rolls is kept at a constant $370 \mathrm{~m} / \mathrm{min}$, the rotational speed of the NR is constant at $5.6 \mathrm{~Hz}$ and that of the WR decreases during the winding process. In addition, the natural frequencies vary because of the variation in physical quantities such as mass and inertia moment of the WR.

Both ends of the rolls are supported by deep-groove ball bearings, and the chock blocks of the NR are pressurized by a pressurizer, which in turn exerts pressure on the thin sheet at a constant load. Vibration occurs during the winding process in a plane that runs through the axis lines of the WR and the NR so that continuous operation becomes impossible, and the quality of the thin sheets is sometimes damaged.

Figures 2(a) to (d) show the frequency analyses of the displacement at the chock block for a NR, and show the results just after the start of the winding process $(H=0 \mathrm{~mm})$ and when $H=12,28$ and $53 \mathrm{~mm}$. The displacement is normalized by the maximum amplitude when $H=0 \mathrm{~mm}$. At the start of the operation, there was no vibration, but when $H$ was about $12 \mathrm{~mm}$, a vibration of $14 \mathrm{~Hz}$ occurred. The vibration then ceased when $H=28 \mathrm{~mm}$, but reappeared, this time at $10 \mathrm{~Hz}$, when $H$ was $53 \mathrm{~mm}$. This appearance and disappearance of vibration is a characteristic of the unstable vibration of a thin sheet winder.

Figure 3 shows the relationship between wound thickness $H$ and vibration frequency when the vibration occurs during the winding operation. The contrasting density represents the amplitude of the vibration, and the level of density shows the magnitude of the amplitude. After the start of the winding operation, an unstable vibration of $14 \mathrm{~Hz}$ (Region A) occurred, which then disappeared. But later, an unstable vibration of $10 \mathrm{~Hz}$ (Region B) occurred. The experiment was stopped at $H=60 \mathrm{~mm}$ because the vibration had become too large. In this paper, the authors clarify the fact that the vibrations of region A and B are pattern formation phenomena caused by different vibration modes, and theoretically analyze

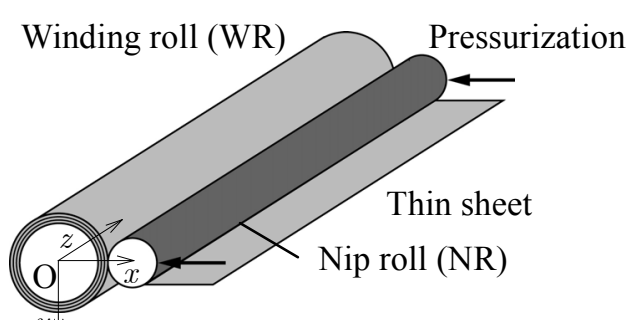

(a) Outline of a thin sheet winder

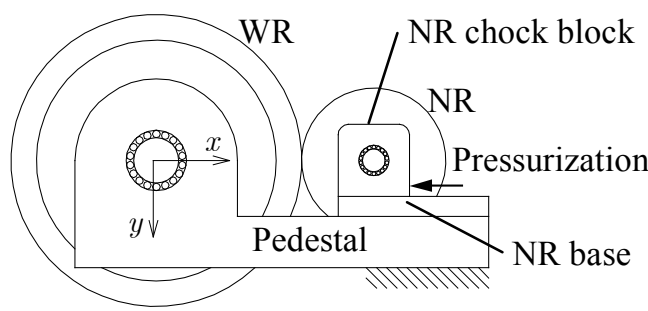

(b) Experimental apparatus

Fig. 1 Outline of a thin sheet winder and experimental apparatus 


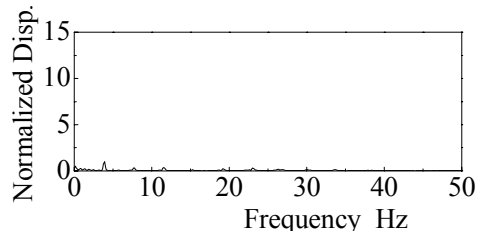

(a) $H=0 \mathrm{~mm}$

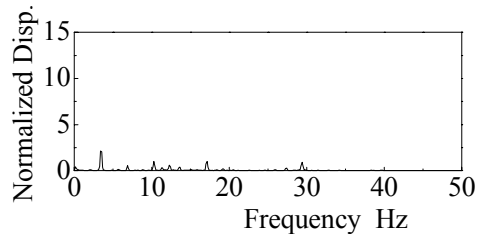

(c) $H=28 \mathrm{~mm}$

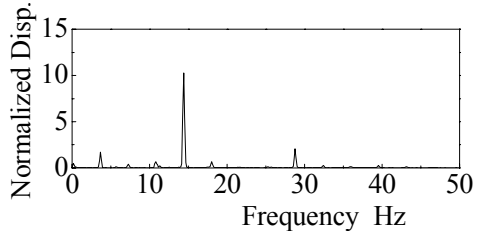

(b) $H=12 \mathrm{~mm}$

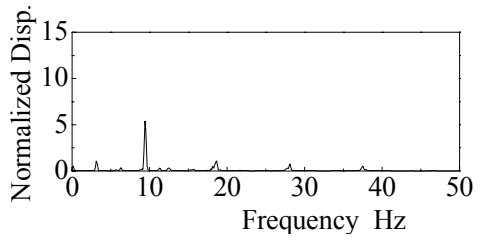

(d) $H=53 \mathrm{~mm}$

Fig. 2 Frequency analysis (Experimental results)

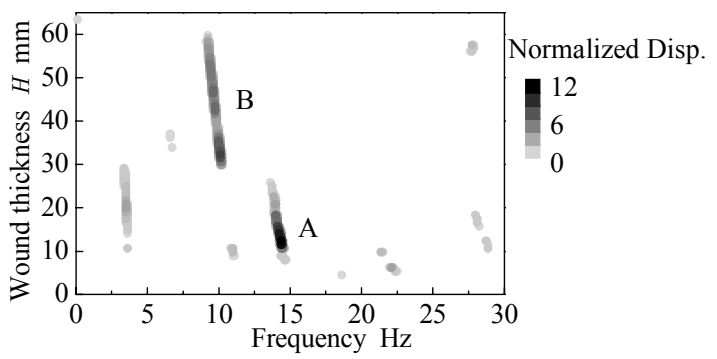

Fig. 3 Vibration frequency and amplitude in operation

the optimum design of dynamic absorbers to suppress the unstable vibrations.

\section{Theoretical Analysis}

As the rubber on the surface of the NR is much harder than the sheet being wound, only the deformation of the wound sheet at the contact part (nip section) is considered. The deformed pattern of the wound sheet generates vibration in the plane that goes through the axis lines of the WR and the NR. Therefore, this system is modeled as a two-layered structure of a lumped mass system, as shown in Fig. 4. The subscript $j$ represents the nodal point $j$, and the superscripts $W$ and $N$ represent the physical quantities related to the WR and the NR, respectively. In some cases, these superscripts $W$ and $N$ are represented together by superscript $R$.

The WR and the NR have the same length $L$, and are divided into $m$ equal elements. The mass and inertia moment of the WR and the NR are divided into the lumped masses on the $m+1$ points (the 0 -th nodal point is the left end of the roll and the $m$-th nodal point is the right end of the roll; the number of nodal points together is $m+1$ ), and the sections between the nodal points are treated as massless beams of length $l(=L / m)$. The masses and inertia moments around $y$ axis of the divided elements are equally distributed at both ends of each element and represented by $m^{R}$ and $J^{R}$, respectively, except for both ends of the rolls. As the chock blocks that support the bearings can only vibrate in the translational direction because of structural constraints, only the increment masses of chock blocks $M^{R}$ are considered, and the increment inertia moments are neglected. The chock blocks are supported by springs and dashpots $\left(K_{h}^{R}, C_{h}^{R}\right)$. Henceforth, the nodal points of both ends are designated by $h(=0$ and $m)$.

The vibration characteristics of the wound sheet at the nip section are modeled by a three-parameter model that can express viscoelastic characteristics in a simple manner. The distributed spring coefficient of the nip section's instantaneous elastic coefficient is expressed by $k^{E}$, and the distributed viscous damping coefficient and spring coefficient of the delayed elastic deformation part are expressed by $c^{V}$ and $k^{V}$, respectively. These are 


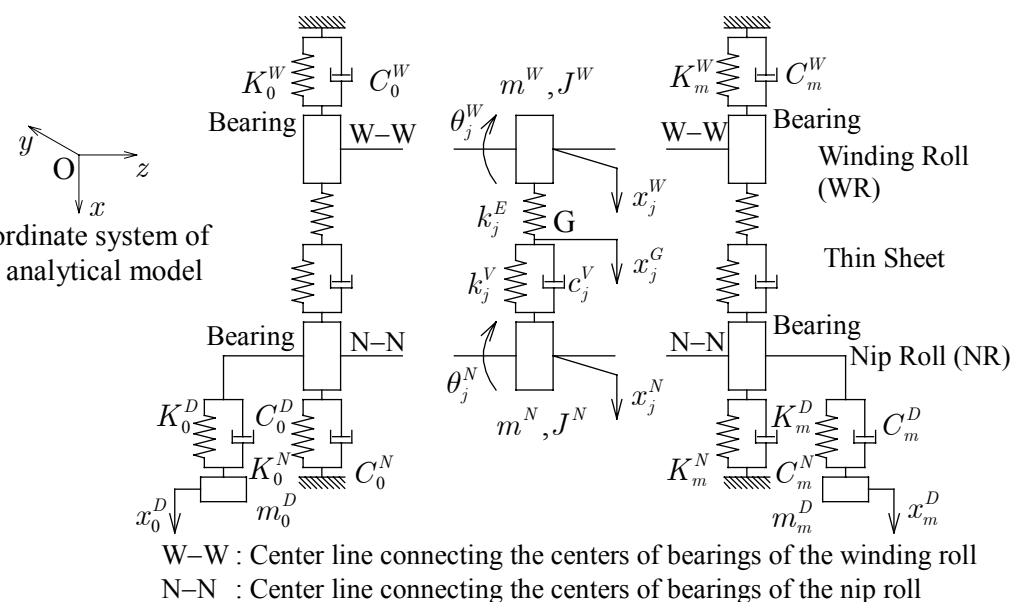

Fig. 4 Analytical model

represented by equivalent concentrated spring $\left(k_{j}^{E}, k_{j}^{V}\right)$ and dashpot $c_{j}^{V}$ at nodal points. An imaginary point between the instantaneous and the delayed spring/dashpot of the three-parameter model is designated as point $\mathrm{G}$, and the displacements of $\mathrm{G}$ at the nodal point $j$ are expressed by $x_{j}^{G}$. The lateral displacements and the rotations around $y$ axis of each roll at the nodal point $j$ are expressed by $x_{j}^{R}$ and $\theta_{j}^{R}$.

As both the generation mechanism of the pattern formation phenomena of the thin sheet and the suppressive effect of the dynamic absorbers are treated in the theoretical analysis, a case in which the dynamic absorbers are attached to the NR chock blocks is analyzed. The dynamic absorbers consist of masses $m_{h}^{D}$, springs $K_{h}^{D}$ and dashpots $C_{h}^{D}$. The displacements of the dynamic absorbers are expressed by $x_{h}^{D}$. In the case of a calculation without dynamic absorbers, $K_{h}^{D}$ and dashpots $C_{h}^{D}$ are equal to zero.

Most of the rubber deformation at the nip section will recover during the time period of the WR rotation $T$, and the residual deformation will be fed back as a forced displacement when the section re-enters the nip section. During the growth process of the pattern formation phenomenon, this deformation is gradually accumulated in the delayed deformation section.

The equations of motion of the WR and the NR in the translational and rotational directions at nodal points $r(=1, \cdots, m-1)$ and $h(=0$ and $m)$, and the equations of motion of the dynamic absorbers at the both ends of the NR are given by,

$$
\begin{aligned}
& \boldsymbol{M}_{r}^{W} \ddot{\boldsymbol{d}}_{r}^{W}+\boldsymbol{k}_{r}^{E}\left(\boldsymbol{d}_{r}^{W}-\boldsymbol{d}_{r}^{G}\right)+\overline{\boldsymbol{f}}_{r}^{W}-\boldsymbol{f}_{r}^{W}=\mathbf{0} \\
& \boldsymbol{M}_{r}^{N} \ddot{\boldsymbol{d}}_{r}^{N}+\boldsymbol{k}_{r}^{E}\left(\boldsymbol{d}_{r}^{G}-\boldsymbol{d}_{r}^{W}\right)+\overline{\boldsymbol{f}}_{r}^{N}-\boldsymbol{f}_{r}^{N}=\mathbf{0} \\
& \boldsymbol{M}_{h}^{W} \ddot{\boldsymbol{d}}_{h}^{W}+\boldsymbol{C}_{h}^{W} \dot{\boldsymbol{d}}_{h}^{W}+\boldsymbol{K}_{h}^{W} \boldsymbol{d}_{h}^{W}+\boldsymbol{k}_{h}^{E}\left(\boldsymbol{d}_{h}^{W}-\boldsymbol{d}_{h}^{G}\right)+\overline{\boldsymbol{f}}_{h}^{W}-\boldsymbol{f}_{h}^{W}=\mathbf{0} \\
& \boldsymbol{M}_{h}^{N} \ddot{\boldsymbol{d}}_{h}^{N}+\boldsymbol{C}_{h}^{N} \dot{\boldsymbol{d}}_{h}^{N}+\boldsymbol{K}_{h}^{N} \boldsymbol{d}_{h}^{N}+\boldsymbol{k}_{h}^{E}\left(\boldsymbol{d}_{h}^{G}-\boldsymbol{d}_{h}^{W}\right) \\
& \quad+\boldsymbol{C}_{h}^{D}\left(\dot{\boldsymbol{d}}_{h}^{N}-\dot{\boldsymbol{d}}_{h}^{D}\right)+\boldsymbol{K}_{h}^{D}\left(\boldsymbol{d}_{h}^{N}-\boldsymbol{d}_{h}^{D}\right)+\overline{\boldsymbol{f}}_{h}^{N}-\boldsymbol{f}_{h}^{N}=\mathbf{0} \\
& m_{h}^{D} \ddot{x}_{h}^{D}+C_{h}^{D}\left(\dot{x}_{h}^{D}-\dot{x}_{h}^{N}\right)+K_{h}^{D}\left(x_{h}^{D}-x_{h}^{N}\right)=0
\end{aligned}
$$

where superscript notation " $\bullet$ " indicates the derivative with respect to time.

$$
\begin{aligned}
& \boldsymbol{M}_{r}^{R}=\operatorname{diag}\left(m^{R}, J^{R}\right), \boldsymbol{M}_{h}^{R}=\operatorname{diag}\left(m^{R} / 2+M^{R}, J^{R} / 2\right), \boldsymbol{M}_{h}^{D}=\operatorname{diag}\left(m_{h}^{D}, 0\right) \\
& \boldsymbol{C}_{h}^{R}=\operatorname{diag}\left(C_{h}^{R}, 0\right), \boldsymbol{K}_{h}^{R}=\operatorname{diag}\left(K_{h}^{R}, 0\right), \boldsymbol{C}_{h}^{D}=\operatorname{diag}\left(C_{h}^{D}, 0\right), \boldsymbol{K}_{h}^{D}=\operatorname{diag}\left(K_{h}^{D}, 0\right) \\
& \boldsymbol{k}_{j}^{E}=\operatorname{diag}\left(k_{j}^{E}, 0\right), k_{r}^{E}=k^{E} l, k_{h}^{E}=k_{r}^{E} / 2 \\
& \boldsymbol{d}_{j}^{R}={ }^{t}\left(x_{j}^{R}, \theta_{j}^{R}\right), \boldsymbol{d}_{j}^{G}={ }^{t}\left(x_{j}^{G}, 0\right), \boldsymbol{d}_{h}^{D}={ }^{t}\left(x_{h}^{D}, 0\right) \\
& \boldsymbol{f}_{j}^{R}={ }^{t}\left(F_{j}^{R}, N_{j}^{R}\right), \overline{\boldsymbol{f}}_{j}^{R}={ }^{t}\left(\bar{F}_{j}^{R}, \bar{N}_{j}^{R}\right), j=0, \cdots, m, \overline{\boldsymbol{f}}_{0}^{R}=\boldsymbol{f}_{m}^{R}=\mathbf{0}
\end{aligned}
$$

The left superscript $t$ indicates transposed representation. $F_{j}^{R}, N_{j}^{R}$ represent share stress and moment, respectively, and the variables with and without head symbol "-" represent the physical quantities on the left and right sides at a node, respectively.

If the flexural rigidity of the equally divided beam elements of length $l$ are $(E I)^{R}$ 
and the influence coefficient of cantilevers of length $l$ are $(\tilde{\alpha}, \tilde{\beta}, \tilde{\gamma})^{R}$ for $i=1, \cdots, m$, from the force balance between both ends of the beam elements, we obtain the following equations:

$$
\begin{aligned}
& \boldsymbol{d}_{i}^{R}={ }^{t} \boldsymbol{L} \boldsymbol{d}_{i-1}^{R}+\boldsymbol{G}^{R} \overline{\boldsymbol{f}}_{i}^{R}, \quad \boldsymbol{f}_{i-1}^{R}=\boldsymbol{L} \overline{\boldsymbol{f}}_{i}^{R} \\
& \boldsymbol{L}=\left(\begin{array}{ll}
1 & 0 \\
l & 1
\end{array}\right), \boldsymbol{G}^{R}=\left(\begin{array}{cc}
\tilde{\alpha} & \tilde{\gamma} \\
\tilde{\gamma} & \tilde{\beta}
\end{array}\right)^{R},\left(\tilde{\alpha}^{R}, \tilde{\beta}^{R}, \tilde{\gamma}^{R}\right)=\left(\frac{l^{3}}{3(E I)^{R}}, \frac{l}{(E I)^{R}}, \frac{l^{2}}{2(E I)^{R}}\right)
\end{aligned}
$$

From the balance of sharing force within the wound sheet element-considering the feedback effects of residual deformation of the wound sheet - the following expression is obtained:

$$
k_{j}^{E}\left\{x_{j}^{G}(t)-x_{j}^{W}(t)\right\}+c_{j}^{V}\left\{\dot{u}_{j}(t)-e^{-\alpha T} \dot{u}_{j}(t-T)\right\}+k_{j}^{V}\left\{u_{j}(t)-e^{-\alpha T} u_{j}(t-T)\right\}=0
$$

where $k_{r}^{V}=k^{V} l, c_{r}^{V}=c^{V} l, k_{h}^{V}=k_{r}^{V} / 2, c_{h}^{V}=c_{r}^{V} / 2, T$ is one revolution period of the WR, $\alpha$ is the recovery-characteristic exponent of the recoverable wound-sheet deformation and is different from $k_{j}^{V} / c_{j}^{V}$, which can be calculated by the three-parameter model of the vibration property at the nip section. $u_{j}(t)$ is the amount of wound-sheet deformation for nodal point $j$ immediately after passing through the nip section, and is expressed by the following equation:

$$
u_{j}(t)=x_{j}^{G}(t)-x_{j}^{N}(t)
$$

In order to let the revolution period of WR be $2 \pi$, the following variable transformation is applied to Eqs. (1), (3) and (5)

$$
\tau=\omega t, \omega=2 \pi / T
$$

After this transformation, Laplace transformation is applied to Eqs. (1), (3) and (5) with all the initial values equating to zero, and some treatments, such as elimination of terms concerning $x_{j}^{G}$ and $x_{h}^{D}$ are performed. Then, we obtain

$$
\boldsymbol{A} \boldsymbol{X}=\mathbf{0}
$$

where $\boldsymbol{A}$ is a square threefold block diagonal matrix of $4(m+1)$-th order, $\boldsymbol{X}$ is a vector of $4(m+1)$ dimension,

$$
\begin{aligned}
& \boldsymbol{A}=\left(\begin{array}{cccccc}
\boldsymbol{P}_{0}+\boldsymbol{Q} & \tilde{\boldsymbol{Q}} & & & & \\
{ }^{t} \tilde{\boldsymbol{Q}} & \boldsymbol{P}_{1}+\overline{\boldsymbol{Q}}+\boldsymbol{Q} & \tilde{\boldsymbol{Q}} & & 0 & \\
& { }^{t} \tilde{\boldsymbol{Q}} & \boldsymbol{P}_{2}+\overline{\boldsymbol{Q}}+\boldsymbol{Q} & \tilde{\boldsymbol{Q}} & & \\
& & & & \ddots & \\
& 0 & & \ddots & \ddots & \tilde{Q} \\
& & & & { }^{t} \tilde{Q} & \boldsymbol{P}_{m}+\overline{\boldsymbol{Q}}
\end{array}\right) \\
& \boldsymbol{X}={ }^{t}\left({ }^{t} \boldsymbol{D}_{0},{ }^{t} \boldsymbol{D}_{1}, \cdots,{ }^{t} \boldsymbol{D}_{m}\right), \boldsymbol{P}_{j}=\left(\begin{array}{cc}
\boldsymbol{P}^{W}+\tilde{\boldsymbol{P}}^{E} & -\tilde{\boldsymbol{P}}^{E} \\
-\tilde{\boldsymbol{P}}^{E} & \boldsymbol{P}^{N}+\tilde{\boldsymbol{P}}^{E}+\boldsymbol{P}^{D}
\end{array}\right)_{j} \\
& \boldsymbol{P}_{r}^{R}=\omega^{2} s^{2} \boldsymbol{M}_{r}^{R}, \boldsymbol{P}_{r}^{D}=\mathbf{0}, \boldsymbol{P}_{h}^{R}=\omega^{2} s^{2} \boldsymbol{M}_{h}^{R}+\omega s \boldsymbol{C}_{h}^{R}+\boldsymbol{K}_{h}^{R} \\
& \tilde{\boldsymbol{P}}_{j}^{E}=\operatorname{diag}\left(k_{j}^{E}-\frac{\left(k_{j}^{E}\right)^{2}}{k_{j}^{E}+\left(\omega s c_{j}^{V}+k_{j}^{V}\right)\left(1-e^{-\alpha T-2 \pi s}\right)}, 0\right) \\
& \boldsymbol{P}_{h}^{D}=\operatorname{diag}\left(\omega s C_{h}^{D}+K_{h}^{D}-\frac{\left(\omega s C_{h}^{D}+K_{h}^{D}\right)^{2}}{\omega^{2} s^{2} m_{h}^{D}+\omega s C_{h}^{D}+K_{h}^{D}}, 0\right), \overline{\boldsymbol{Q}}=\operatorname{Diag}\left(\boldsymbol{H}^{W}, \boldsymbol{H}^{N}\right) \\
& \tilde{\boldsymbol{Q}}=-\operatorname{Diag}\left(\boldsymbol{L} \boldsymbol{H}^{W}, \boldsymbol{L} \boldsymbol{H}^{N}\right), \boldsymbol{Q}=\operatorname{Diag}\left(\boldsymbol{L} \boldsymbol{H}^{W t} \boldsymbol{L}, \boldsymbol{L} \boldsymbol{H}^{N t} \boldsymbol{L}\right), \boldsymbol{H}^{R}=\left(\boldsymbol{G}^{R}\right)^{-1}
\end{aligned}
$$

$\boldsymbol{D}_{j}$ is an image function after a Laplace transformation of vector ${ }^{t}\left({ }^{t} \boldsymbol{d}^{W},{ }^{t} \boldsymbol{d}^{N}\right)_{j}$, Diag is a block diagonal matrix, and $s$ is a variable of the Laplace transformation.

From Eq. (8), the characteristic equation becomes

$\operatorname{det} \boldsymbol{A}=0$

If one of these characteristic roots, which satisfy Eq. (10), has a positive real part, then the system becomes unstable, and a pattern formation phenomenon starts to occur.

As the system treated here is a lumped mass system and the degree of freedom is very 
large, it is readily anticipated that the computation of characteristic roots requires considerable computation time. Therefore, in order to compute Eq. (10) efficiently, the transfer influence coefficient method developed by the authors is introduced, and the left side of Eq. (10) is transformed as

$$
\operatorname{det} \boldsymbol{A}=\frac{\operatorname{det} \boldsymbol{P}_{0}}{(\operatorname{det} \boldsymbol{G})^{m}} \prod_{i=1}^{m} \operatorname{det}\left(\boldsymbol{I}+\overline{\boldsymbol{T}}_{i} \boldsymbol{P}_{i}\right)
$$

where $\boldsymbol{G}=\operatorname{Diag}\left(\boldsymbol{G}^{W}, \boldsymbol{G}^{N}\right), \boldsymbol{I}$ is the 4 th order unit matrix, and with $\boldsymbol{U}=\operatorname{Diag}(\boldsymbol{L}, \boldsymbol{L})$, $\boldsymbol{T}_{0}=\left(\boldsymbol{P}_{0}\right)^{-1}$

$$
\overline{\boldsymbol{T}}_{i}={ }^{t} \boldsymbol{U} \boldsymbol{T}_{i-1} \boldsymbol{U}+\boldsymbol{G}, \quad\left(\boldsymbol{I}+\overline{\boldsymbol{T}}_{i} \boldsymbol{P}_{i}\right) \boldsymbol{T}_{i}=\overline{\boldsymbol{T}}_{i} .
$$

Thus the computation of Eq. (11) is carried out recurrently by numerical computation of Eq. (12), which contains four unknown simultaneous equations. That is, computation of Eq. (10), which consists of a square threefold block diagonal matrix of $4(m+1)$-th order, is transformed to the computation of $m$ simultaneous algebraic equations of four unknowns. In the computation, the derivatives of $\operatorname{det} \boldsymbol{A}$ are obtained by numerical difference, and the characteristic roots are determined by applying the Newton-Raphson method to Eq. (11).

\section{Results of Numerical Calculation and Discussions}

\subsection{Standard parameters and natural frequencies}

The support stiffness and the characteristics of the nip section are shown in Table 1. The weights of the moving parts of the WR without a wound sheet, and the NR are $2400 \mathrm{~kg}$ and $430 \mathrm{~kg}$, respectively. The mass of the WR when the wound thickness is $H$ is given by

$$
M_{H}=2400+\pi \rho H\left(2 r_{W R}+H\right)
$$

where $r_{W R}$ is radius of the WR and $\rho$ is the density of the wound sheet.

In Table 1, stiffness is decided by the natural frequencies of the experimental apparatus and the vibration frequency of the unstable vibrations. The viscoelastic characteristics of the thin sheet were decided by referencing the data of the rubber and the elastic yarn ${ }^{(2),(4)}$. These values are assumed to be constant during operation, because the target of the analysis is around the wound thicknesses in which the unstable vibrations in Fig. 3 occur. As it was difficult to decide the damping of the support element by experiment, it is assumed that the damping ratio of each roll, which is assumed to be a rigid body, is constant at 0.05 . As the viscoelastic characteristics of the thin sheet were remarkable, the recovery characteristic exponent was set to $\alpha=0.1 \mathrm{~s}^{-1}$. The division number $m$ is set to 20 .

First, a case in which dynamic absorbers are not fitted is analyzed to clarify the mechanism of the pattern formation phenomena in the sheet-winding process. Figure 5 shows the relationship among wound thickness $H$ and $q$-th coupled natural frequency $f_{q}$ and the rotating speed of the WR, $f$. Figure 6 shows the natural modes when $H=53$ $\mathrm{mm}$. The upper and lower lines represent the natural mode of the WR and the NR, respectively. The dotted lines show the centers of the vibration modes. The results up to the 3rd mode are shown in Fig. 6. The natural frequency of the 3rd mode decreases as the wound thickness increases. However, the natural frequencies of the 1st and 2nd modes are almost constant, even though the wound thickness increases. This is because the NR mainly vibrates, and the increase in the wound thickness does not have an affect on the natural

Table 1 Standard parameters

\begin{tabular}{ccc}
\hline Parameters & Value & Units \\
\hline$K_{0}^{W}, K_{m}^{W}$ & 30 & $\mathrm{MN} / \mathrm{m}$ \\
$K_{0}^{N}, K_{m}^{N}$ & 0.45 & $\mathrm{MN} / \mathrm{m}$ \\
$k^{E}$ & 0.11 & $\mathrm{MN} / \mathrm{m}^{2}$ \\
$k^{V}$ & 0.8 & $\mathrm{MN} / \mathrm{m}^{2}$ \\
$c^{V}$ & 0.14 & $\mathrm{kNs} / \mathrm{m}^{2}$ \\
$\alpha$ & 0.1 & $\mathrm{~s}^{-1}$ \\
\hline
\end{tabular}




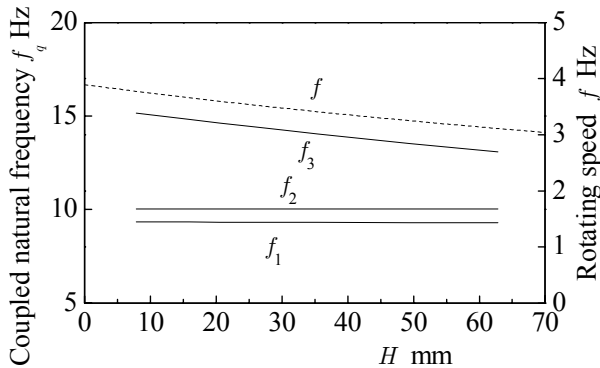

Fig. 5 Coupled natural frequencies $f_{q}$ and rotating speed $f$

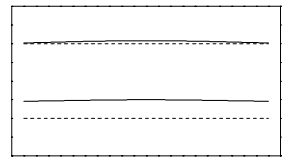

1st mode

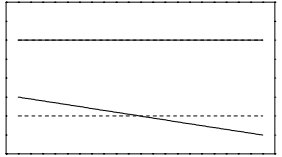

2nd mode

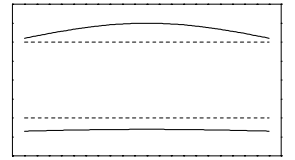

3rd mode

Fig. 6 Natural modes at $H=53 \mathrm{~mm}$

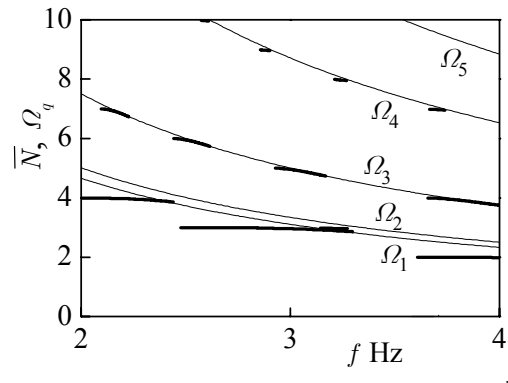

Fig. 7 Relationship between $f$ and $\bar{N}$ ( $H=12 \mathrm{~mm})$

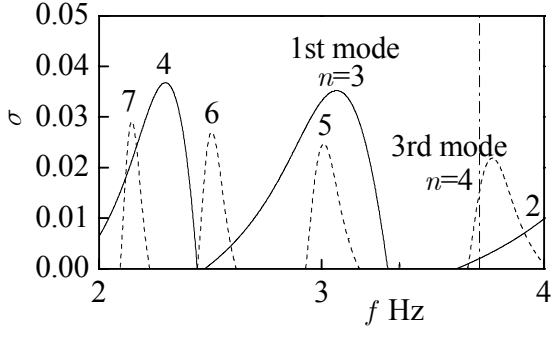

Fig. 8 Relationship between $f$ and $\sigma$ ( $H=12 \mathrm{~mm})$

frequencies in these natural modes.

\subsection{Analysis of pattern formation phenomena}

Figure 7 shows the relationship between the rotating speed of the WR, $f$ and the imaginary part of the characteristic root $\bar{N}(=\operatorname{Im}(s))$ when $H=12 \mathrm{~mm}$ and dynamic absorbers are not fitted. The hyperbolas $\Omega_{q}\left(=f_{q} / f\right)$ indicate the ratio of the $q$-th natural frequency $f_{q}$ to the WR rotation frequency $f$. Solid thick lines indicate the loci of unstable characteristic roots. The results of $q=1, \cdots, 5$ are shown in Fig. 7 . From the figure, it can be seen that the unstable regions exist around the natural frequencies of the 1st to the 4th modes, and the imaginary part of the characteristic root $\bar{N}$ exists near integer. The unstable regions of the 1st and 3rd modes are the largest of all. Figure 8 shows the relationship between the rotating speed of WR, $f$ and the real part of the characteristic root $\sigma$ of the 1 st and 3rd modes when the same parameter as in Fig. 7 is used. The solid lines and dotted lines indicate the results of the 1 st and $3 \mathrm{rd}$ modes, respectively. The dashed line indicates the rotating speed of WR when $H=12 \mathrm{~mm}$. The values of $\sigma$ of the 2 nd and 4 th modes are smaller than those of the 1 st and 3 rd modes. The maximum value of $\sigma$ of the 2 nd and 4 th modes is about $10 \%$ of the 1 st and 3 rd modes, so the instability of the 2 nd and 4 th modes is less than that of the 1 st and 3rd modes. Henceforth, the 1 st and 3rd modes are regarded as a target for analysis. In the case of the pattern formation phenomena with viscoelastic deformation, the imaginary part of the characteristic root $\bar{N}$ is less than integer $^{(7)}$. For the sake of convenience, the number $n$ that is shown in each locus expresses the nearest integer to $\bar{N}(n \geq \bar{N})$, and we call this the polygonal number. From the figure, the dashed line intersects with $n=4$ of the $3 \mathrm{rd}$ mode and $n=2$ of the 1 st mode, so $n=4$ of the 3 rd mode is supposed to occur in this wound thickness, because its real part $\sigma$ is larger than that of the 1 st mode. 


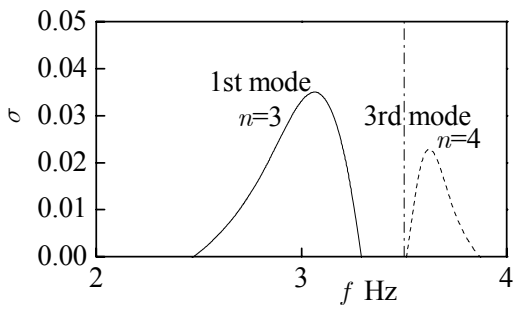

(a) $H=28 \mathrm{~mm}$

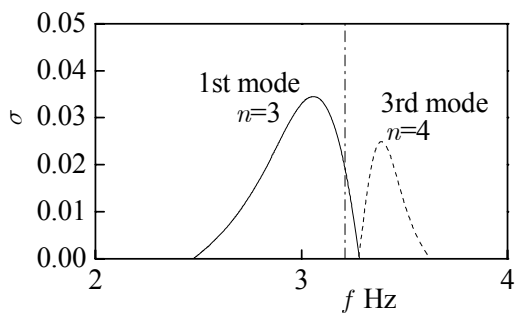

(b) $H=53 \mathrm{~mm}$

Fig. 9 Relationship between $f$ and $\sigma$

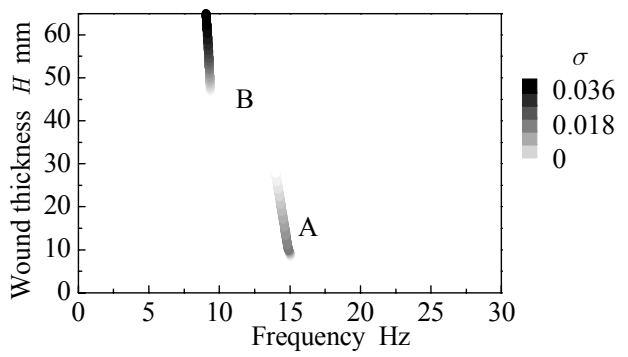

Fig. 10 Relationship between vibration frequency and $\sigma$ in operation

Figures 9(a) and 9(b) show the loci of the instability $\sigma$ when $H=28 \mathrm{~mm}$ and 53 $\mathrm{mm}$, respectively. Only the unstable regions of $n=3$ of the 1 st mode and $n=4$ of the 3rd mode are indicated. The other regions are omitted because they exist far from the rotating speed of the WR. Henceforth, only the 1st and the 3rd modes are considered. From Figs. 9(a) and 9(b), the unstable region of the 3rd mode moves to the lower frequency side, because the 3rd mode natural frequency decreases with the increase of the wound thickness. On the other hand, the unstable region of the 1st mode does not move, because the natural frequency of the 1st mode is almost constant (see Fig. 5). From Figs. 8 and 9, it is found that the pattern formation of $n=4$ of the 3 rd mode occurs when the wound thickness is small, and then the unstable region disappears, but after that the pattern formation of $n=3$ of the 1st mode occurs.

Figure 10 shows the relationship between the vibration frequency and the wound thickness $H$. The contrasting density shows the magnitude of the real part of the characteristic roots $\sigma$. The level of density shows the level of instability. When the wound thickness is small, the viscoelastic characteristics at the nip section are strongly related to the characteristics of the rubber covering the NR. For this reason, when the wound thickness $H$ is smaller than $10 \mathrm{~mm}$, we assume the values $\bar{k}^{E}, \bar{k}^{V}, \bar{\alpha}$, which are the substitute for $k^{E}, k^{V}, \alpha$, as the following functions:

$$
\begin{aligned}
& \bar{k}^{E}=k^{E}(1.2 H+13), \bar{k}^{V}=7 \bar{k}^{E} \\
& \bar{\alpha}=\left(\alpha^{\prime}-\alpha\right)(H / 10)^{2}-2\left(\alpha^{\prime}-\alpha\right)(H / 10)+\alpha^{\prime}
\end{aligned}
$$

In this equation, the functions of $\bar{k}^{E}, \bar{k}^{V}$ are assumed by considering the longitudinal elastic modulus ratio of the rubber to the thin sheet, and by referencing the characteristics of the rubber in previous papers ${ }^{(2),(4)}$. The function $\bar{\alpha}$ is assumed to be the same value of rubber $\alpha^{\prime}\left(=2 \mathrm{~s}^{-1}\right)$ in the previous papers ${ }^{(2),(4)}$ when the wound thickness is equal to zero.

Although Fig. 3, which shows the amplitude of the pattern formation, is not comparable to Fig. 10, which shows the instability $\sigma$ directly, the computational results yield a good agreement with the experimental results in the transition from regions $\mathrm{A}$ to $\mathrm{B}$, the vibration frequencies and the distribution range of the unstable regions.

\subsection{Optimum design of dynamic absorbers}

To confirm the suppressive effect of dynamic absorbers on the pattern formation phenomena of a thin sheet winder, the numerical calculation is performed in a case where dynamic absorbers are fitted to the chock blocks of the NR. Considering the long length of 


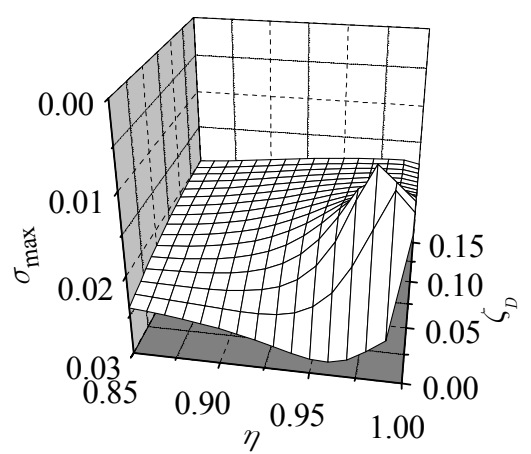

(a) $H=12 \mathrm{~mm}$ (Dynamic absorbers for the 3rd mode)

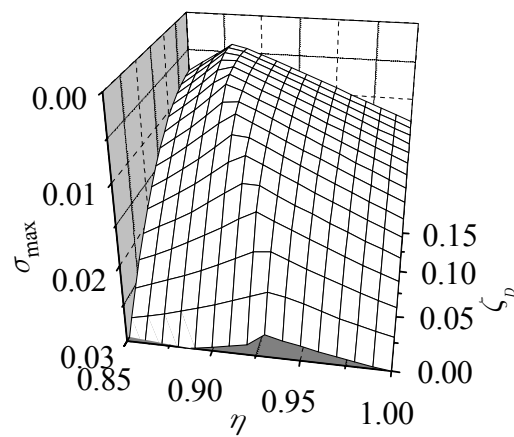

(b) $H=53 \mathrm{~mm}$ (Dynamic absorbers for the 1st mode)

Fig. 11 Optimum design of dynamic absorbers

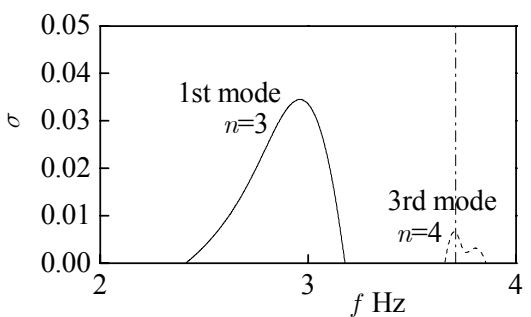

(a) $H=12 \mathrm{~mm}$ (Dynamic absorbers for the 3rd mode)

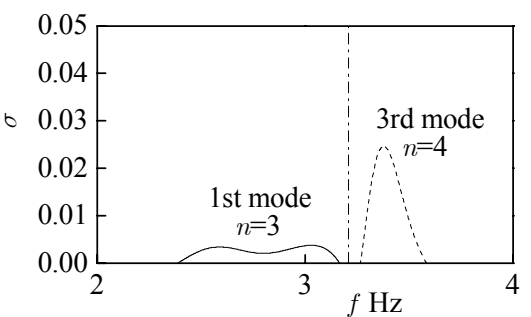

(b) $H=53 \mathrm{~mm}$ (Dynamic absorbers for the 1 st mode)

Fig. 12 Unstable regions when two dynamic absorbers are fitted

the roll, two dynamic absorbers that have the same characteristics are fitted to the chock blocks of the NR. The mass ratio of a dynamic absorber to the NR is $\mu=0.025$. The optimum design of dynamic absorbers to suppress the unstable regions of the 3rd mode's $n=4$ in $H=12 \mathrm{~mm}$ and the 1st mode's $n=3$ in $H=53 \mathrm{~mm}$ was examined by changing the value of the natural frequency $f_{D}$ and the damping ratio $\zeta_{D}\left(=C_{h}^{D} / 2 \sqrt{m_{h}^{D} K_{h}^{D}}\right)$ of the dynamic absorbers. The instability of an unstable region is evaluated by the maximum value of the characteristic roots $\sigma_{\max }$ of this region.

Figures 11(a) and 11(b) show $\sigma_{\max }$ of the 3rd mode's $n=4$ in $H=12 \mathrm{~mm}$ and the 1st mode's $n=3$ in $H=53 \mathrm{~mm}$, respectively. The abscissa represents the ratio of the natural frequency of the dynamic absorbers to that of the targeted mode of suppression $\eta\left(=f_{D} / f_{q}\right)$, and the ordinate represents the damping ratio of the dynamic absorbers $\zeta_{D}$. From the figures, the optimum value to suppress the pattern formation phenomena can be seen for each mode. Although the dynamic absorbers of $\mu=0.025$ cannot suppress pattern formation totally $(\sigma<0)$, dynamic absorbers with $\eta=0.98$ and $\zeta_{D}=0.02$ are the optimum design for the $3 \mathrm{rd}$ mode in $H=12 \mathrm{~mm}$, and dynamic absorbers with $\eta=0.88$ and $\zeta_{D}=0.14$ are the optimum design for the 1 st mode in $H=53 \mathrm{~mm}$. The values of $\sigma_{\max }$ in Figures 8 and 9 (b) are reduced to about $1 / 2$ and 1/5, respectively. This means that the time to reach a level of amplitude is 2 times and 5 times as long, respectively as when there are no dynamic absorbers fitted.

\subsection{Suppression of pattern formation by dynamic absorbers}

The performance of the optimized dynamic absorbers in suppressing the pattern formation phenomena is evaluated. Henceforth, we call the optimized dynamic absorbers in the wound thickness $H=12 \mathrm{~mm}$ and $H=53 \mathrm{~mm}$ the optimum dynamic absorbers for the $3 \mathrm{rd}$ mode and the 1st mode, respectively. As the natural frequency of the 1st mode is almost constant throughout the winding operation, the natural frequency of the optimum dynamic absorbers for the 1st mode remains constant, which is designed in $H=53 \mathrm{~mm}$. On the other hand, as the natural frequency of the 3rd mode decreases during operation, the frequency ratio $\eta$ of the optimum dynamic absorbers for the 3rd mode remains constant. 


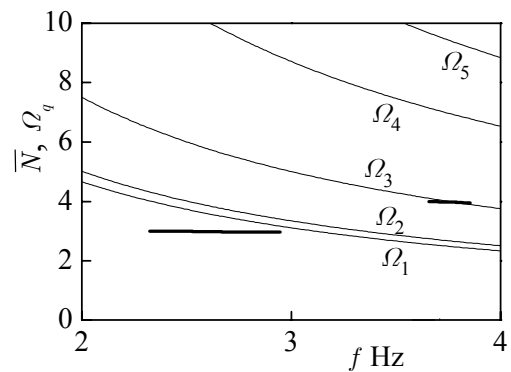

(a) Relationship between $f$ and $\bar{N}(H=12 \mathrm{~mm})$

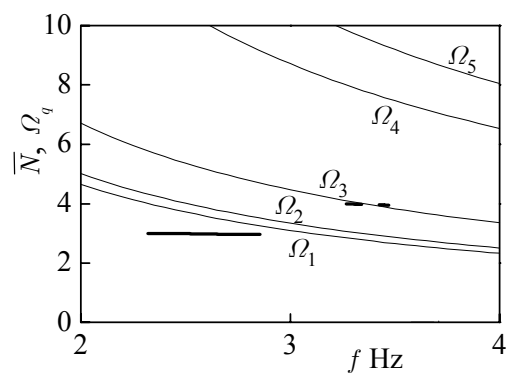

(c) Relationship between $f$ and $\bar{N}(H=53 \mathrm{~mm})$

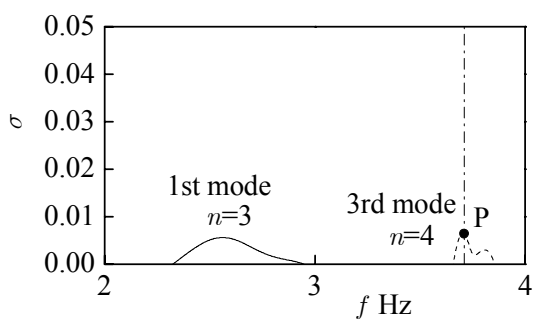

(b) Relationship between $f$ and $\sigma(H=12 \mathrm{~mm})$

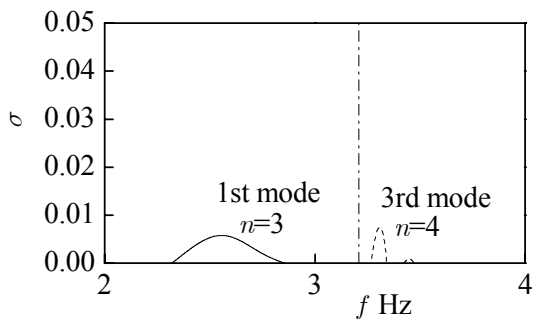

(d) Relationship between $f$ and $\sigma(H=53 \mathrm{~mm})$

Fig. 13 Unstable region when 4 dynamic absorbers are fitted.

This means that the natural frequency of the optimum dynamic absorbers for the 3rd mode varies during the winding operation. Figures 12(a) and 12(b) show the unstable regions in a case where optimum dynamic absorbers for the 3 rd and the 1st modes are fitted to the NR chock blocks, respectively. Compared with Figs. 8 and 9(b), the instability is reduced by attaching the optimum dynamic absorbers in the operating range. The instability of modes that are not the target of suppression is not changed by fitting dynamic absorbers.

\subsection{Suppression of pattern formation by using $\mathbf{4}$ dynamic absorbers}

In the numerical computation shown above, two dynamic absorbers that have the same characteristics are fitted to the chock blocks of the NR to suppress a mode. Thus, if the dynamic absorbers maintain the effect of suppression during operation, the characteristics of the dynamics absorbers should be changed to match the change of the natural frequencies and the vibration modes. Moreover, near the range around $f=3 \mathrm{~Hz}$ in Fig.8, the unstable regions of $n=3$ of the 1 st mode and $n=5$ of the 3 rd mode exist together, and the vibration frequencies of these modes are different. This means that it is impossible to suppress pattern formations by using only one type of dynamic absorber. Therefore, in order to solve this problem and to reduce instability over a wide range of operating speeds, the optimum design of four dynamic absorbers, two for the 1st mode and two for the 3rd mode, is investigated.

The amendment of the theoretical analysis when the four dynamic absorbers are fitted is just the addition of $\boldsymbol{P}_{h}^{D}$ for the dynamic absorbers in Eq. (9). Figures 13(a) and 13(b) show the relationship between $f$ and $\bar{N}$ and the relationship between $f$ and $\sigma$ in a case where two dynamic absorbers for the 3rd mode optimized in $H=12 \mathrm{~mm}$ and two dynamic absorbers for the 1st mode optimized in $H=53 \mathrm{~mm}$, are attached to the chock blocks when $H=12 \mathrm{~mm}$, respectively. The mass ratio of a dynamic absorber to the weight of the NR is $\mu=0.025$. Even though the natural frequency of the optimum dynamic absorbers for the 1st mode, which is designed in $H=53 \mathrm{~mm}$, is constant, the unstable regions are suppressed. This is because the natural frequency of the 1st mode is almost constant throughout operation. That is, in order to suppress the pattern formation phenomena of the 1st mode, fitting dynamic absorbers that are optimally designed in a wound thickness to the chock blocks is effective. According to the complex mode at point $\mathrm{P}$ 


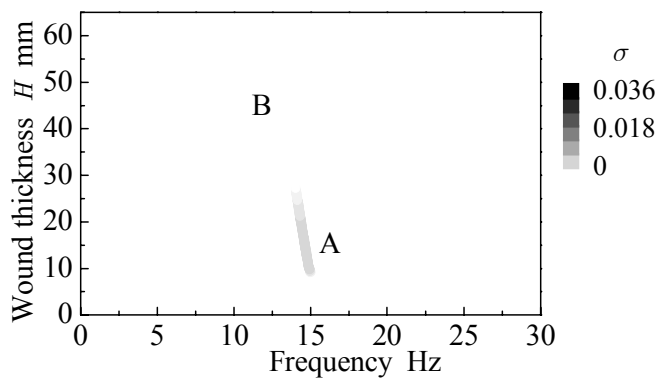

Fig. 14 Relationship between vibration frequency and $\sigma$ in operation when 4 dynamic absorbers are fitted

in Fig. 13(b), the amplitude ratios of the dynamic absorbers for the 1st and the 3rd modes to the center of the NR are about 0.2 and 10, respectively, and the phases of the dynamic absorbers for the 1 st and the 3rd modes based on the center of the NR are almost out-of-phase.

Figures 13(c) and 13(d) show the relationship between $f$ and $\bar{N}$ and the relationship between $f$ and $\sigma$ in a case where two dynamic absorbers for the 3 rd mode in the optimum conditions in Fig. 11(a) $\left(\eta=0.98, \zeta_{D}=0.02\right)$ and two optimum dynamic absorbers for the 1st mode that is optimized in $H=53 \mathrm{~mm}$ are attached to the chock blocks when $H=53 \mathrm{~mm}$, respectively. The unstable region of the 1st mode shrinks as a result of fitting optimum dynamic absorbers for the 3rd mode. Even though the optimum dynamic absorbers for the 3rd mode are designed in $H=12 \mathrm{~mm}$, the unstable region of the $3 \mathrm{rd}$ mode is suppressed in this wound thickness. That is, the optimum condition of the dynamic absorbers does not vary during operation. However, as the natural frequency of the 3rd mode varies with the wound thickness, the natural frequency of the dynamic absorbers should vary to maintain this condition $\eta=0.98$. Tunable dynamic absorbers offer a good solution to this problem (see Sec. 5). Consequently, the pattern formation phenomena can be suppressed by using four dynamic absorbers at any rotating speed during the entire winding process.

Figure 14 shows the result obtained in the same manner as Fig. 10 for a case in which two optimum dynamic absorbers for the 1st mode, and two dynamic absorbers for the 3rd mode are fitted to the NR chock blocks. The instability of region A is greatly suppressed, and unstable region B disappears in the wound thickness range in this figure. The unstable region of the 1st mode does not appear until the wound thickness $H$ equals $100 \mathrm{~mm}$.

As the optimum condition of the dynamic absorber required in this paper is one to reduce the maximum value of instability in a locus of the unstable region, this is not equal to a design to reduce the instability at a certain rotating speed. Furthermore, the mass of a dynamic absorber is decided by the ratio of the dynamic absorber to the weight of the NR, and the mass of the dynamic absorber is very small compared to the overall weight of the system. Considering these conditions, further stabilization or the perfect suppression of the pattern formation can be realized.

\subsection{Proposition for the structure of a tunable dynamic absorber}

In the previous section, in order to suppress the 3rd mode's pattern formation phenomena during operation in its entirety, it was demonstrated that a tunable dynamic absorber is useful. Thus, although an experiment has not been conducted, an example of structure of a tunable dynamic absorber is introduced in this section.

Figures 15(a) and 15(b) show an example of a tunable dynamic absorber and a detailed view, respectively. This dynamic absorber is composed of a mass, a base, bearings, two beams, and two gears. The cross sections of the beams $\mathrm{P}$ and $\mathrm{Q}$ are rectangle, and the mass is attached to the beam in between $\mathrm{P}$ and $\mathrm{Q}$ via the bearings. Both ends of two beams are attached to the base via bearings. The gears are fixed on to the ends of the shafts. The gears 


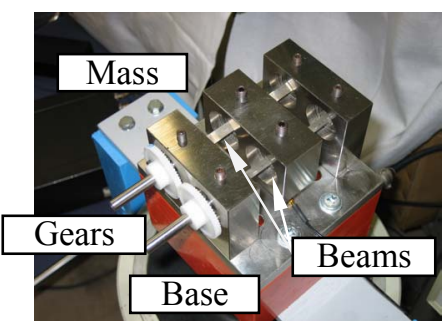

(a) Tunable dynamic absorber

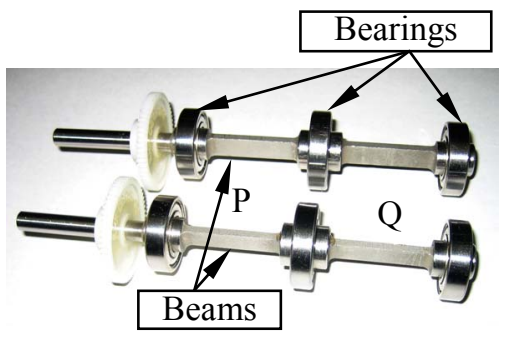

(b) Detailed view

Fig. 15 Structure of a tunable dynamic absorber

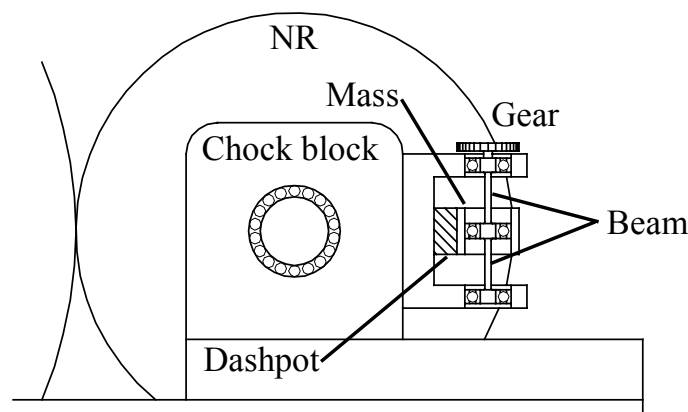

Fig.16 Tunable dynamic absorbers attached to the NR chock block

rotate the shafts at the same angle in opposite directions. As the angle of rotation increases, the geometric moment of inertia varies continuously without changing the direction of the principal axis. The angle of rotation is controlled by a motor. As the stiffness of the beams that support the mass vary continuously due to the rotation of the shafts, the natural frequency also varies continuously.

The mass or the support position moves in a conventional tunable dynamic absorber ${ }^{(8),(9)}$. The main features of the proposed new tunable absorber are its compact structure and the tuning accuracy achieved by changing the shape of the cross section. If the part from beam Q to the end of the shaft is omitted, it becomes an overhang-type tunable absorber.

Figure 16 shows the sample structure when the proposed tunable dynamic absorber is attached to the NR chock block. The base of the dynamic absorber is fixed on the chock block. The damping element is set between the mass and the base. In a case where four dynamic absorbers need to be attached to suppress the pattern-formation phenomena, two dynamic absorbers can be positioned on the chock block in parallel on each side. If the cross section of the beam is assumed to be a rectangle having $b_{D} \times h_{D}\left(b_{D}<h_{D}\right)$, the geometric moment of inertia varies from $b_{D} h_{D}{ }^{3} / 12$ to $h_{D} b_{D}{ }^{3} / 12$ continuously, and also the natural frequency of the dynamic absorber varies continuously. The value of the damping should be set to the optimum damping ratio of the targeted mode.

\section{Conclusions}

The authors conducted an experiment using an experimental machine to reproduce the pattern formation phenomena in thin sheet winders. The authors also theoretically analyzed the phenomena as a time-lag system due to viscoelastic deformation of the wound sheet, and clarified the generation mechanism and proposed the countermeasure by using dynamic absorbers. The results obtained are summarized as follows:

(1) During the winding process of thin sheet, the unstable vibration mode changed from the 3 rd mode to the 1 st mode as a result of a change in parameters, including rotating speed and mass. The analytical results showed a good agreement with the phenomena generated in the experimental apparatus.

(2) From the computational results for a case in which dynamic absorbers were fitted to the chock blocks of the nip roll, it was found that there are optimum values of the natural frequency and the damping ratio of the dynamic absorbers to suppress the 
pattern formation phenomena. The optimum value for each mode is different. The pattern formation phenomena were suppressed by using two identical dynamic absorbers, whose mass is about $2.5 \%$ that of the nip roll. In order to suppress the pattern formation phenomena from occurring during the winding process, the natural frequency and the damping ratio of the dynamic absorbers need to be adjusted to the optimum values for each mode.

(3) By installing two dynamic absorbers for the 1st and 3rd modes (four in total), the instability of both modes could be reduced. In this case, the natural frequency for the dynamic absorbers for the 3rd mode needed to be changed to suppress the pattern formation phenomena throughout the entire winding process.

(4) The fundamental design of a novel tunable dynamic absorber that would suppress the pattern formation phenomena was proposed.

(5) An optimum design to reduce instability at a certain rotating speed and the mass effect of the dynamic absorber on the pattern formation phenomena are subjects for future work.

\section{References}

(1) Roisum, D.R., Winder Vibration Can Reduce Operating Efficiency and Increase Maintenance Costs, Tappi Journal, Vol.71,No.1, (1981), pp.87-96.

(2) Sueoka, A. et al., Pattern Formation Generated in a Winder System of Textile Machine, JSME Int. J., Series C, Vol.41,No.3, (1998), pp.630-638.

(3) Crandall, S.H. and Pariseanu, G., Limit-Cycle Vibrations of a Rolling Cylinder, International Journal of Non-Linear Mechanics, Vol.20, No.5/6, (1985), pp.385-393.

(4) Sueoka, A. et al., Pattern Formation of Roll-Covering Rubber in a Paper-Making Machine and Its Countermeasures (in Japanese), Trans. JSME, Series C, Vol.61,No.591, (1995), pp. $4160-4167$.

(5) Matsuzaki, K. et al., Polygonal Wear of Work Rolls in a Hot Leveler of Steel Making Machine (3rd Report, A Countermeasure by Using Dynamic Absorbers) (in Japanese), Trans. JSME, Series C, Vol.71,No.704, (2005), pp.1123-1130.

(6) Kondou, T. et al., Optimum Design of Dynamic Absorber for Pattern Formation Phenomena Generated in Contact Rotating Systems (1st Report, In Case of Single Degree-of Freedom System) (in Japanese), Trans. JSME, Series C, Vol.71,No.704, (2005), pp.1131-1138.

(7) Sueoka, A. et al., Polygonal Deformation of Roll-Covering Rubber, JSME Int. J., Series C, Vol.39,No.1, (1996), pp.1-10.

(8) Nagaya, K. et al., Vibration Control of a Structure by Using a Tunable Absorber and an Optimal Vibration Absorber under Auto-Tuning Control, Journal of Sound and Vibration, 228(4), (1999), pp.773-792.

(9) Seto, K., Effect of a Variable Stiffness-Type Dynamic Absorber on Improving the Performance of Machine-Tools with a Long Overhung Ram, Annals of the CIRP, Vol.27,No.1, (1978), pp.327-332. 